\title{
Heavy Metal Determination by Sequential Extraction in Agricultural Farmland Soils In Selected Farms In Abraka, Delta State, Nigeria
}

\author{
*Agbaire, P. O and Akporhonor, E. E \\ Chemistry Department Delta State University, Abraka
}

\begin{abstract}
This study investigated the speciation of heavy metals ( $\mathrm{Cd}, \mathrm{Cu}, \mathrm{Pb}, \mathrm{Mn}, \mathrm{Co}, \mathrm{Ni}$, and $\mathrm{Zn}$ ) in agricultural farmland soils in Abraka, Delta State, Southern, Nigeria. Soil's physicochemical parameters were also determined with the following results. $\mathrm{pH}$ ranged from 4.20-5.45(acidic soil), total organic carbon from 0.95-2.02\%. Particle size showed soil to be mainly sandy, percentage nitrogen from 0.18-0.35 while phosphorus from 21.11-58.33mg/Kg, Cation Exchange Capacity ranged from 1.04-7.96Cmol/Kg. The percentage of the studied heavy metals in the various fraction are in the following order; $C d$ : Residual >acid soluble > oxidizable $>$ reducible >exchangeable. $\mathrm{Cu}$ : Reducible > acid soluble > residual > oxidizable > exchangeable. Pb: Residual > reducible > acid soluble > oxidizable > exchangeable. Mn: Residual > Exchangeable > acid soluble > reducible > oxidizable. Co: Acid soluble > reducible > residual > oxidizable > exchangeable. Ni: Reducible > oxidizable > acid soluble > exchangeable > residual. Zn: Acid soluble >oxidizable > residual > reducible > exchangeable. Exchangeable $\mathrm{Cd}$, Co, and $\mathrm{Pb}$ were not detected. The absence of mobile form of $\mathrm{Cd}$, $\mathrm{Co}$ and $\mathrm{Pb}$ in all the soil samples eliminates the toxic risk both to the tropic chain and from its migration downwards the soil profile. The heavy metals speciation of the soil samples indicates that the heavy metals were predominately in the non-residual fractions suggestive of control by anthropogenic sources. The mean total extracted metals are Cd: 1.09-1.28, Cu: 8.82-11.46, Pb: 1.51-1.69, Mn: 58.29-64.86, Co, 0.88-1.32, Ni: 6.457.97, $\mathrm{Zn:} 15.78-17.43 \mathrm{mg} / \mathrm{Kg}$.
\end{abstract}

Keywords: Heavy metals, Speciation, Agricultural farmland Soils, Abraka, Delta State, Nigeria

\section{Introduction}

Soils are the reservoir for many materials; beneficial and harmful. These include biological chemical as well as heavy metals. Total metal content of soils is useful for most geochemical works but often the speciation (bioavailability) of these metals are more important to the environmentalist. Speciation is defined according to Tack and Verloo (1995) as "the identification and quantification of different defined species, forms, or phases in which an element occurs". Heavy metals on the other hand refer to any metallic element that has a relatively high density and is toxic even at low concentration (Lenntech, 2004, Duruibe et al., 2007, Uba et al., 2009). The accumulation of heavy metals in soil poses risk to human and the ecosystem's health (Afshin and Masaud, 2008, Nwachukwu et al., 2010, Odoh, 2011). Risks may be passed to human either through the food chain or through direct exposure. Risks arising from pollution of soils by heavy metals are well known (Nriagu, 1990). In recent years extensive research has been conducted on the estimation of bioavailability and toxicity of metals in soils (Ferguson 1990). Currently no methods are available that allows accurate prediction of plant intake or phytotoxicty, adverse effects on human or ecotoxicity from metal pollution of soils (Odoh 2011). It is widely accepted that to assess the environmental input of soil pollution, the determination of metals speciation will give more information about the potential for release of contaminants and subsequent toxicity. Therefore, in environmental studies of risk assessment, chemical partitioning among the various phases is more useful than the measurement of total heavy metal content (Uba et al., 2008, Albores et al., 2000, Odoh et al., 2011, Osakwe 2010). Among the procedures to determine elemental speciation is that of Tessier et al. 1979. There is no information on speciation of metals in the agricultural farmland soils in Abraka, Delta State, Nigeria, despite it is one of the leading producers of Garri (processed cassava tuber meal) in this area. This work was therefore undertaken to assess the heavy metal load of the farmland soil using sequential extraction method of Tessier et al. 1979. The villages identified are Agbarha (AG), Urhuoka (UR), Umeghe (UM), Urhovie (UV), Erho (ER), Urhuogbessa (US), Oria (OR), Ugono (UG), and Otorho (OT). These areas are purely agricultural in nature. The metals investigated include $\mathrm{Cd}, \mathrm{Cu}, \mathrm{Mn}, \mathrm{Pb}, \mathrm{Ni}, \mathrm{Co}$, and $\mathrm{Zn}$. These metals were selected because of their importance in agriculture as micronutrients $(\mathrm{Co}, \mathrm{Cu}, \mathrm{Mn}$, and $\mathrm{Zn})$ and also because of their toxicity ( $\mathrm{Cd}, \mathrm{Ni}$, and $\mathrm{Pb})$ 


\section{Sampling Sites}

\section{Materials And Methods}

Abraka is a University town located in Ethiope East Local Government Area of Delta State, Southern Nigeria. It lies between latitude $5^{\circ} 46^{1} 54^{11} \mathrm{~N}$ and longitude $6^{\circ} 05^{1} 59^{11} \mathrm{E}$. It has a lowland type of landscape which is grouped under the coastal lowlands of Western Nigeria within the tropical rainforest region.

A total of nine sites of eighteen samples were collected with nine top- soil $(0-15 \mathrm{~cm})$ and nine sub- soils (15$30 \mathrm{~cm}$ ). A control site with similar ecological features but has not been farmed was also selected (around the one of the University student hostels).

Composite soil samples were collected and air dried for weeks to get rid of the excess moisture. The dried soil samples were then crushed in a porcelain mortar with a pestle. They were then sieved through a $2 \mathrm{~mm}$ sieve made of stainless steel for analysis of soil $\mathrm{pH}$ and particle size. Some portion of the individual sieved soil sample were further pulverized to a fine powder and passed through a $0.5 \mathrm{~mm}$ sieve for analysis of organic carbon, total metal content and speciation. The soil samples were then stored in well labelled polyethylene bags ready for analysis.

The $\mathrm{pH}$ of the soil in deionized water sample 1:1 (Tan, 1996) was determined by a portable pH meter (model HI 98107) according to standard analytical procedures. Organic matter was determined using chromic acid oxidation method (Walkley and Black 1934). Particle size distribution was determined by the hydrometer method (Bougucos 1951). The exchangeable cation was determined by the method according to Jackson, 1958.

\section{Sequential Extraction of Metals}

The extraction was carried out with an initial mass of $1.0 \mathrm{~g}$ of air dried soil samples in polypropylene centrifuge tube of $50 \mathrm{~cm}^{3}$ capacities. The Tessier et al., method of 1979 was employed with the replacement of $\mathrm{MgCl}_{2}$ with $\mathrm{Mg}\left(\mathrm{NO}_{3}\right)_{2}$ to avoid an increase in the solubility of the heavy metals within the soil solution matrix. The extractions were carried out as follows;

Exchangeable phase (F1); Soil samples were extracted with $8 \mathrm{~cm}^{3}$ of $1 \mathrm{~m} \mathrm{Mg}\left(\mathrm{NO}_{3}\right)_{2}$ at a pH of 7 for 1 hour with continuous agitation.

The Carbonate Bound (F2); The residue from F1 was extracted with $8 \mathrm{~cm}^{3}$ of $1 \mathrm{~m} \mathrm{NaOAc}$ solution (adjusted to $\mathrm{pH} 5$ with $\mathrm{HOAc}$ ) for a period of 5 hours at room temperature with continuous agitation.

Fe-Mn Oxide Bound (Reducible) (F3); The residue from F2 was extracted with $10 \mathrm{~cm}^{3}$ of $0.04 \mathrm{~m}$ hydroxylamine chloride $\left(\mathrm{NH}_{2} \mathrm{OH} . \mathrm{HCl}\right)$ in $25 \% \mathrm{HOAc}$ for 6 hours at $96^{\circ} \mathrm{C}$ with occasional agitation.

Organically Bound ( Oxidizable) (F4); The residue from F3 was extracted with $8 \mathrm{~cm}^{3}$ of $30 \% \mathrm{H}_{2} \mathrm{O}_{2}$ (adjusted to $\mathrm{pH} 2$ with $\mathrm{HNO}_{3}$ ) for 30min. in a boiling water bath and the supernatant was filtered after centrifuging. This step was repeated twice. The residue from the above step was washed three times with $5 \mathrm{~cm}^{3}$ of $3.2 \mathrm{mNH}_{4} \mathrm{OAc}$ solutions in $20 \% \mathrm{v} / \mathrm{v}$ nitric acid $\left(\mathrm{HNO}_{3}\right)$, for $30 \mathrm{~min}$. with continuous agitation.

Residual F5; the residual from F4 was quantitatively transferred into a beaker, evaporated to dryness and digested with $\mathrm{HNO}_{3}-\mathrm{H}_{2} \mathrm{O}_{2}-\mathrm{HCl}$ mixture using a water bath reflux digestion.

After each successive extraction separation was done by centrifuging for 30mins the supernatants were removed with a pipette and then filtered. The residue was then washed with $8 \mathrm{~cm}^{3}$ of deionised water, followed by vigorous hand shaking. It was then centrifuged for 30mins before the next extraction. The F1, F2 and F3 were individually made up to $25 \mathrm{~cm}^{3}$ in a volumetric flask while the filtrate from F4 and F5 were made up to $50 \mathrm{~cm}^{3}$ in a volumetric flask.

QUALITY ASSURANCE: All reagents used were of analytical grade. All quality assurance procedures in the laboratory were carefully adhered to.

STATISTICAL ANALYSIS: Determination of heavy metal mobility is based on the relative amount of the metal in the exchangeable fraction (F1) containing the mobile form and the carbonate bound fraction (F2) containing the easily mobilizable phases. The mobility index for metals MF was expressed as based on the Kabala and Singh equation;

$$
\begin{aligned}
& \mathrm{MF}=\underline{\mathrm{F} 1+\mathrm{F} 2} \quad \mathrm{X} 100 \% \\
& \mathrm{~F} 1+\mathrm{F} 2+\mathrm{F} 3+\mathrm{F} 4+\mathrm{F} 5
\end{aligned}
$$




\section{Results And Discussion}

Table 1: Soil Characterisation of Abraka Agricultural Farmland

\begin{tabular}{|c|c|c|c|c|c|c|c|c|c|}
\hline $\begin{array}{l}\text { Sampling } \\
\text { Sites }\end{array}$ & $\mathrm{pH}$ & $\%$ Clay & $\%$ Sand & $\%$ Silt & $\begin{array}{l}\% \text { Org } \\
\text { Matter }\end{array}$ & $\% \mathrm{~N}$ & Total P & Avail. P & $\begin{array}{l}\text { Conductivity } \\
\mathrm{Us} / \mathrm{Cm}\end{array}$ \\
\hline $\begin{array}{l}\text { Agbarha } \\
\text { (AG) }\end{array}$ & $4.40 \pm 0.42$ & $2.76 \pm 0.41$ & $94.47 \pm 6.21$ & $4.26 \pm 0.52$ & $1.33 \pm 0.40$ & $0.35 \pm 0.10$ & 91.11 & 1 & \pm 1.85 \\
\hline $\begin{array}{l}\text { Urhuoka } \\
\text { (UR) }\end{array}$ & $4.20 \pm 0.14$ & $0.5 \pm 0.70$ & $95.37 \pm 3.34$ & $4.14 \pm 0.34$ & $1.23 \pm 0.46$ & $0.25 \pm 0.05$ & $146.66 \pm 6.60$ & $58.33 \pm 1.96$ & $48.60 \pm 2.06$ \\
\hline Umeghe(UM) & $4.65 \pm 0.21$ & $6.52 \pm 0.11$ & $87.24 \pm 0.84$ & $6.24 \pm 0.50$ & $1.80 \pm 0.93$ & $0.25 \pm 0.05$ & $94.45 \pm 4.72$ & $23.89 \pm 2.50$ & $121.8 \pm 6.02$ \\
\hline Ugono (UG) & $5.21 \pm 0.01$ & $5.74 \pm 1.53$ & $93.10 \pm 0.11$ & $1.16 \pm 0.25$ & $0.95 \pm 0.33$ & $0.32 \pm 0.05$ & $98.89 \pm 4.72$ & $37.78 \pm 2.28$ & $63.40 \pm 1.38$ \\
\hline Otorho (OT) & $4.45 \pm 0.21$ & $4.55 \pm 1.14$ & $91.05 \pm 6.23$ & $4.40 \pm 0.21$ & $1.51 \pm 0.79$ & $0.18 \pm 0.05$ & $71.12 \pm 5.28$ & $23.33 \pm 1.57$ & $34.20 \pm 3.11$ \\
\hline Urhuovie & $5.45 \pm 0.35$ & $2.28 \pm 1.22$ & $92.45 \pm 1.06$ & $5.27 \pm 0.35$ & $1.74 \pm 0.99$ & $0.18 \pm 0.05$ & $95.55 \pm 4.28$ & $32.23 \pm 2.72$ & $65.10 \pm 1.01$ \\
\hline Erho (ER) & $4.25 \pm 0.21$ & $0.50 \pm 0.01$ & $95.37 \pm 1.24$ & $4.13 \pm 0.21$ & $1.68 \pm 0.37$ & $0.28 \pm 0.01$ & $70.00 \pm 7.87$ & $16.67 \pm 4.72$ & $48.80 \pm 9.05$ \\
\hline (US) & $5.30 \pm 0.28$ & $4.59 \pm 1.53$ & $91.88 \pm 2.65$ & $5.30 \pm 0.28$ & $2.02 \pm 1.24$ & $0.34 \pm 0.08$ & $91.11 \pm 3.14$ & $35.56 \pm 9.43$ & $142.1 \pm 31.25$ \\
\hline Oria (OR) & $4.25 \pm 0.35$ & $6.11 \pm 1.50$ & $89.69 \pm 4.71$ & $4.20 \pm 0.35$ & $1.65 \pm 0.39$ & $0.25 \pm 0.04$ & $97.77 \pm 2.01$ & $40.00 \pm 4.28$ & $46.6 \pm 6.33$ \\
\hline
\end{tabular}

TABLE 2; Mean \pm SD of Exchangeable Cations in Abraka Agricultural Farmland soils

$\begin{array}{llllll}\text { Sampling Sites } & \mathrm{Na} & \mathrm{K} & \mathrm{Mg} & \mathrm{Ca} & \mathrm{CEC} \\ \text { Agbarha (AG) } & 0.16 & 0.3 & 0.56 & 0.03 & 1.05 \\ \text { Urhuoka (UR) } & 0.44 & 0.74 & 0.87 & 0.03 & 2.08 \\ \text { Umeghe (UM) } & 0.02 & 0.11 & 2.09 & 0.09 & 2.31 \\ \text { Ugono (UG) } & 0.05 & 0.07 & 5.07 & 0.11 & 5.30 \\ \text { Otorho (OT) } & 0.06 & 0.07 & 2.45 & 0.06 & 2.64 \\ \text { Urhuovie (UV) } & 0.07 & 0.16 & 3.06 & 0.20 & 3.49 \\ \text { Erho (ER) } & 0.11 & 0.21 & 0.87 & 0.10 & 1.29 \\ \text { Urhuagbessa (US) } & 0.47 & 0.54 & 6.88 & 0.07 & 7.96\end{array}$

TABLE 3: Cd Concentration in each fraction of Abraka Agricultural Farmland soils

$\begin{array}{llllllllll} & \mathrm{AG} & \mathrm{UR} & \mathrm{UM} & \mathrm{UG} & \mathrm{OT} & \mathrm{UV} & \mathrm{ER} & \mathrm{US} & \mathrm{OR} \\ \text { Exchangeable } & \mathrm{ND} & \mathrm{ND} & \mathrm{ND} & \mathrm{ND} & \mathrm{ND} & \mathrm{ND} & \mathrm{ND} & \mathrm{ND} & \mathrm{ND} \\ \text { Oxidizable } & 0.24 \pm 0.01 & 0.22 \pm 0.10 & 0.22 \pm 0.10 & 0.21 \pm 0.05 & 0.24 \pm 0.11 & 0.23 \pm 0.07 & 0.18 \pm 0.02 & 0.23 \pm 0.03 & 0.22 \pm 0.02 \\ \text { Acid soluble } & 0.40 \pm 0.01 & 0.38 \pm 0.12 & 0.44 \pm 0.11 & 0.34 \pm 0.05 & 0.21 \pm 0.12 & 0.42 \pm 0.05 & 0.36 \pm 0.03 & 0.30 \pm 0.04 & 0.35 \pm 0.04 \\ \text { Reducible } & 0.22 \pm 0.12 & 0.20 \pm 0.11 & 0.18 \pm 0.05 & 0.16 \pm 0.01 & 0.21 \pm 0.06 & 0.22 \pm 0.05 & 0.24 \pm 0.03 & 0.22 \pm 0.06 & 0.21 \pm 0.04 \\ \text { Residual } & 0.41 \pm 0.11 & 0.38 \pm 0.10 & 0.44 \pm 0.06 & 0.42 \pm 0.04 & 0.43 \pm 0.05 & 0.39 \pm 0.06 & 0.40 \pm 0.04 & 0.42 \pm 0.05 & 0.39 \pm 0.06 \\ \text { \{extracted } & 1.27 & 1.18 & 1.28 & 1.13 & 1.09 & 1.26 & 1.18 & 1.17 & 1.17 \\ \text { Metals } & 67.72 & 67.8 & 65.62 & 62.83 & 60.55 & 69.05 & 66.60 & 64.10 & 66.67 \\ \text { Non-residual \% } & 32.28 & 32.2 & 34.38 & 37.17 & 39.45 & 30.95 & 33.40 & 35.90 & 33.33 \\ \text { Residual \% } & 18.90 & 18.64 & 17.19 & 18.58 & 22.01 & 18.25 & 15.25 & 19.66 & 18.80\end{array}$

$\begin{array}{llllll}\text { Oria }(\mathrm{OR}) & 0.08 & 0.13 & 0.97 & 0.08 & 1.26\end{array}$


TABLE 4: Cu Concentration in each fraction of Abraka Agricultural Farmland soils

\begin{tabular}{|c|c|c|c|c|c|c|c|c|c|}
\hline & $\mathrm{AG}$ & UR & UM & UG & OT & UV & ER & US & OR \\
\hline & $0.50 \pm 0.0$ & & $0.55 \pm 0.1$ & $0.65 \pm 0.1$ & $0.84 \pm 0.1$ & $0.88 \pm 0.1$ & $1.50 \pm 0.1$ & $0.45 \pm 0.1$ & $0.65 \pm 0$ \\
\hline \multirow[t]{2}{*}{ Exchangeable } & 2 & $0.65 \pm 012$ & 2 & 1 & 2 & 1 & 0 & 5 & 2 \\
\hline & $1.73 \pm 0.1$ & & $2.00 \pm 0.0$ & $1.05 \pm 0.0$ & $1.11 \pm 0.1$ & $2.15 \pm 0.1$ & $2.66 \pm 0.1$ & $1.44 \pm 0.1$ & $1.24 \pm 0$ \\
\hline \multirow[t]{2}{*}{ Oxidizable } & 1 & $2.55 \pm 011$ & 7 & 5 & 1 & 0 & 1 & 1 & 4 \\
\hline & $3.30 \pm 0.0$ & $1.42 \pm 0.1$ & $2.52 \pm 0.2$ & $3.44 \pm 0.5$ & $2.42 \pm 0.1$ & $1.65 \pm 0.0$ & $3.55 \pm 0.1$ & $3.88 \pm 0.1$ & $2.75 \pm 0$ \\
\hline \multirow[t]{2}{*}{ Acid soluble } & 3 & 4 & 1 & 0 & 0 & 5 & 0 & 2 & 4 \\
\hline & $2.50 \pm 0.1$ & $4.00 \pm 0.2$ & $3.54 \pm 0.0$ & $2.55 \pm 0.6$ & $3.00 \pm 0.5$ & $2.35 \pm 0.1$ & $2.33 \pm 0.1$ & $3.24 \pm 0.1$ & $3.25 \pm 0$ \\
\hline \multirow[t]{2}{*}{ Reducible } & 2 & 0 & 5 & 0 & 0 & 1 & 0 & 2 & 5 \\
\hline & $1.44 \pm 0.1$ & $2.44 \pm 0.2$ & & $1.33 \pm 0.6$ & $1.45 \pm 0.2$ & $1.44 \pm 0.1$ & $2.12 \pm 0.1$ & $2.14 \pm 0.0$ & $1.11 \pm 0$ \\
\hline \multirow{3}{*}{$\begin{array}{l}\text { Residual } \\
\{\quad \text { Extracted } \\
\text { Metals }\end{array}$} & 1 & 1 & $2.85 \pm 030$ & 0 & 2 & 2 & 2 & 5 & 4 \\
\hline & & & & & & & & & \\
\hline & 9.47 & 11.06 & 11.46 & 9.02 & 8.82 & 8.47 & 12.16 & 11.15 & 9.00 \\
\hline Non-residual \% & 84.79 & 77.94 & 75.51 & 82.25 & 83.56 & 83.00 & 82.57 & 80.81 & 87.67 \\
\hline Residual \% & 15.21 & 22.06 & 24.49 & 14.75 & 16.44 & 17.00 & 17.43 & 19.19 & 12.33 \\
\hline Mobile phase\% & 23.55 & 28.93 & 22.25 & 18.87 & 22.10 & 35.77 & 34.21 & 16.95 & 21.00 \\
\hline
\end{tabular}

TABLE 5: Pb Concentration in each fraction of Abraka Agricultural Farmland Soils

\begin{tabular}{|c|c|c|c|c|c|c|c|c|c|}
\hline & $\mathrm{AG}$ & UR & $\mathrm{UM}$ & UG & OT & UV & ER & US & OR \\
\hline Exchangeable & ND & ND & ND & ND & ND & ND & ND & ND & ND \\
\hline Oxidizable & $0.24 \pm 0.01$ & $0.31 \pm 0.01$ & $0.22 \pm 0.02$ & $0.26 \pm 0.01$ & $0.21 \pm 0.01$ & $0.22 \pm 0.01$ & $0.33 \pm 0.01$ & $0.23 \pm 0.01$ & $0.20 \pm 0.01$ \\
\hline Acid soluble & $0.32 \pm 0.01$ & $0.24 \pm 0.01$ & $0.32 \pm 0.01$ & $0.24 \pm 0.01$ & $0.22 \pm 0.02$ & $0.26 \pm 0.01$ & $0.30 \pm 0.01$ & $0.30 \pm 0.01$ & $0.33 \pm 0.01$ \\
\hline Reducible & $0.45 \pm 0.01$ & $0.47 \pm 0.04$ & $0.44 \pm 0.02$ & $0.54 \pm 0.01$ & $0.46 \pm 0.01$ & $0.45 \pm 0.01$ & $0.46 \pm 0.01$ & $0.47 \pm 0.02$ & $0.44 \pm 0.01$ \\
\hline $\begin{array}{l}\text { Residual } \\
\{\quad \text { Extracted }\end{array}$ & $0.65 \pm 0.11$ & $0.67 \pm 0.02$ & $0.66 \pm 0.01$ & $0.65 \pm 0.01$ & $0.62 \pm 0.01$ & $0.62 \pm 0.02$ & $0.65 \pm 0.01$ & $0.64 \pm 0.01$ & $0.65 \pm 0.02$ \\
\hline Metals & 1.66 & 1.69 & 1.64 & 1.69 & 1.51 & 1.55 & 1.74 & 1.64 & 1.62 \\
\hline Non-residual $\%$ & 60.84 & 60.36 & 59.76 & 61.54 & 58.94 & 60 & 62.64 & 60.98 & 59.88 \\
\hline Residual \% & 39.16 & 39.64 & 40.24 & 38.46 & 41.06 & 40 & 37.36 & 39.02 & 40.12 \\
\hline Mobile phase $\%$ & 14.45 & 18.34 & 13.41 & 15.39 & 13.91 & 14.19 & 18.96 & 14.02 & 12.35 \\
\hline
\end{tabular}

TABLE 6: Mn Concentration in each fraction of Abraka Agricultural Farmland Soils

\begin{tabular}{|c|c|c|c|c|c|c|c|c|c|}
\hline & $\mathrm{AG}$ & UR & UM & UG & OT & UV & ER & US & OR \\
\hline Exchangeable & $10.04 \pm 0.15$ & $11.55 \pm 0.50$ & $10.40 \pm 0.11$ & $10.57 \pm 0.17$ & $11.65 \pm 0.45$ & $11.76 \pm 0.23$ & $12.55 \pm 0.52$ & $10.85 \pm 0.05$ & $11.73 \pm 0.21$ \\
\hline Oxidizable & $7.77 \pm 0.20$ & $8.95 \pm 0.51$ & $7.36 \pm 0.21$ & $9.96 \pm 0.21$ & $8.35 \pm 0.40$ & $9.45 \pm 0.33$ & $9.66 \pm 0.55$ & $9.44 \pm 0.51$ & $9.86 \pm 0.20$ \\
\hline Acid soluble & $10.36 \pm 0.20$ & $9.50 \pm 0.12$ & $11.38 \pm 0.22$ & $12.11 \pm 0.41$ & $10.12 \pm 0.40$ & $11.05 \pm 0.33$ & $10.32 \pm 0.34$ & $11.38 \pm 0.05$ & $10.48 \pm 0.50$ \\
\hline Reducible & $9.46 \pm 0.05$ & $11.44 \pm 0.51$ & $9.47 \pm 0.21$ & $10.35 \pm 0.50$ & $10.14 \pm 0.56$ & $10.47 \pm 0.43$ & $7.86 \pm 0.044$ & $8.77 \pm 0.11$ & $9.66 \pm 0.22$ \\
\hline $\begin{array}{l}\text { Residual } \\
\{\quad \text { Extracted }\end{array}$ & $20.66 \pm 0.15$ & $19.55 \pm 0.52$ & $20.12 \pm 0.22$ & $21.63 \pm 0.55$ & $19.75 \pm 0.33$ & $22.13 \pm 0.56$ & $21.26 \pm 0.05$ & $23.2 \pm 0.51$ & $20.11 \pm 0$ \\
\hline Metals & 58.29 & 60.99 & 58.74 & 64.62 & 60.01 & 64.86 & 61.65 & 63.64 & 61.84 \\
\hline Non-residual $\%$ & 64.56 & 67.95 & 65.75 & 66.53 & 67.09 & 65.88 & 65.52 & 63.54 & 67.48 \\
\hline Residual \% & 35.44 & 32.05 & 34.25 & 33.47 & 32.91 & 34.12 & 34.48 & 36.46 & 32.52 \\
\hline Mobile phase $\%$ & 30.55 & 33.61 & 30.23 & 31.77 & 33.33 & 32.7 & 36.03 & 31.88 & 34.91 \\
\hline
\end{tabular}


TABLE 7: Co Concentration in each fraction of Abraka Agricultural Farmland Soils

$\begin{array}{llllllllll} & \text { AG } & \text { UR } & \text { UM } & \text { UG } & \text { OT } & \text { UV } & \text { ER } & \text { US } & \text { OR } \\ \text { Exchangeable } & \text { ND } & \text { ND } & \text { ND } & \text { ND } & \text { ND } & \text { ND } & \text { ND } & \text { ND } & \text { ND } \\ \text { Oxidizable } & 0.12 \pm 0.01 & 0.14 \pm 0.01 & 0.15 \pm 0.02 & 0.11 \pm 0.01 & 0.15 \pm 0.01 & 0.17 \pm 0.01 & 0.18 \pm 0.01 & 0.19 \pm 0.01 & 0.15 \pm 0.0 \\ \text { Acid soluble } & 0.53 \pm 0.01 & 0.35 \pm 0.02 & 0.30 \pm 0.01 & 0.26 \pm 0.01 & 0.45 \pm 0.02 & 0.34 \pm 0.02 & 0.33 \pm 0.02 & 0.33 \pm 0.02 & 0.36 \pm 0.0 \\ \text { Reducible } & 0.18 \pm 0.02 & 0.34 \pm 0.02 & 0.19 \pm 0.01 & 0.28 \pm 0.01 & 0.30 \pm 0.01 & 0.20 \pm 0.01 & 0.38 \pm 0.02 & 0.32 \pm 0.01 & 0.25 \pm 0.0 \\ \begin{array}{l}\text { Residual } \\ \text { Extracted }\end{array} & 0.22 \pm 0.02 & 0.21 \pm 0.01 & 0.24 \pm 0.02 & 0.24 \pm 0.02 & 0.21 \pm 0.01 & 0.25 \pm 0.02 & 0.43 \pm 0.01 & 0.28 \pm 0.01 & 0.30 \pm 0.0 \\ \begin{array}{l}\text { Metals } \\ \text { Non-residual }\end{array} & 1.05 & 1.04 & 0.88 & 0.89 & 1.11 & 0.96 & 1.32 & 1.12 & 1.06 \\ \begin{array}{l}\text { R } \\ \text { Residual \% }\end{array} & 79.05 & 79.81 & 72.73 & 73.03 & 81.08 & 73.96 & 67.42 & 75.00 & 71.7 \\ \begin{array}{l}\text { Mobile } \\ \text { phase\% }\end{array} & 11.42 & 13.46 & 17.05 & 12.36 & 13.51 & 17.71 & 13.64 & 16.96\end{array}$

TABLE 8: Ni Concentration in each fraction of Abraka Agricutural Farmland Soils

\begin{tabular}{|c|c|c|c|c|c|c|c|c|c|}
\hline & $\mathrm{AG}$ & UR & UM & UG & OT & UV & ER & US & OR \\
\hline Exchangeable & $1.02 \pm 0.01$ & $1.12 \pm 0.05$ & $1.05 \pm 0.01$ & $1.22 \pm 0.01$ & $1.07 \pm 0.01$ & $1.24 \pm 0.01$ & $1.12 \pm 0.01$ & $1.28 \pm 0.01$ & $1.16 \pm 0.10$ \\
\hline Oxidizable & $1.00 \pm 0.02$ & $1.34 \pm 0.01$ & $1.26 \pm 0.02$ & $1.46 \pm 0.05$ & $1.33 \pm 0.11$ & $1.43 \pm 0.02$ & $1.55 \pm 0.01$ & $1.87 \pm 0.11$ & $1.08 \pm 0.11$ \\
\hline Acid soluble & $1.62 \pm 0.02$ & $0.98 \pm 0.01$ & $1.50 \pm 0.01$ & $1.04 \pm 0.02$ & $1.28 \pm 0.10$ & $1.24 \pm 0.01$ & $1.43 \pm 0.02$ & $1.23 \pm 0.11$ & $1.28 \pm 0.02$ \\
\hline Reducible & $2.43 \pm 0.02$ & $2.33 \pm 0.04$ & $2.45 \pm 0.03$ & $2.26 \pm 0.02$ & $2.26 \pm 0.15$ & $2.56 \pm 0.03$ & $2.76 \pm 0.01$ & $2.29 \pm 0.20$ & $2.30 \pm 0.15$ \\
\hline Metals & 7.08 & 6.45 & 7.51 & 7.33 & 6.9 & 7.45 & 7.97 & 7.74 & 6.87 \\
\hline Non-residual \% & 85.73 & 89.46 & 83.36 & 81.58 & 86.09 & 86.85 & 86.07 & 86.18 & 84.72 \\
\hline Residual \% & 14.27 & 10.54 & 16.64 & 18.42 & 13.91 & 13.15 & 13.93 & 13.82 & 15.28 \\
\hline Mobile phase $\%$ & 28.53 & 38.14 & 30.76 & 36.56 & 34.78 & 35.84 & 33.5 & 40.7 & 32.61 \\
\hline
\end{tabular}

TABLE 9: Zn Concentration in each fraction of Abraka Agricultural Farmland Soils

\begin{tabular}{|c|c|c|c|c|c|c|c|c|c|}
\hline & $\mathrm{AG}$ & UR & UM & UG & OT & UV & ER & US & OR \\
\hline & $1.35 \pm 0.1$ & $1.43 \pm 0.1$ & $1.34 \pm 0.5$ & $1.22 \pm 0.4$ & $1.65 \pm 0.0$ & $1.26 \pm 0.1$ & $1.54 \pm 0.1$ & $1.44 \pm 00.1$ & $1.34 \pm 0.1$ \\
\hline \multirow[t]{2}{*}{ Exchangeable } & 5 & 1 & 0 & 4 & 1 & 1 & 5 & 1 & 1 \\
\hline & $4.22 \pm 0.1$ & $3.56 \pm 0.1$ & $3.45 \pm 0.4$ & $4.65 \pm 0.5$ & $3.98 \pm 0.0$ & $3.67 \pm 0.1$ & $3.56 \pm 0.2$ & & $3.55 \pm 0.1$ \\
\hline \multirow[t]{2}{*}{ Oxidizable } & 1 & 1 & 0 & 0 & 4 & 3 & 0 & $3.85 \pm 0.61$ & 4 \\
\hline & $4.21 \pm 0.1$ & $4.45 \pm 0.1$ & $4.22 \pm 0.1$ & $4.67 \pm 0.3$ & $4.34 \pm 0.0$ & $4.48 \pm 0.1$ & $4.34 \pm 0.2$ & & $4.12 \pm 0.5$ \\
\hline \multirow[t]{2}{*}{ Acid soluble } & 5 & 0 & 0 & 4 & 3 & 0 & 1 & $4.89 \pm 0.50$ & 4 \\
\hline & $3.42 \pm 0.1$ & $3.44 \pm 0.1$ & $3.32 \pm 0.5$ & $3.45 \pm 0.3$ & $3.33 \pm 0.0$ & $3.42 \pm 0.1$ & $3.44 \pm 0.1$ & & $3.32 \pm 0.5$ \\
\hline \multirow[t]{2}{*}{ Reducible } & 0 & 2 & 1 & 2 & 1 & 1 & 1 & $3.38 \pm 0.55$ & 5 \\
\hline & $3.12 \pm 0.5$ & $3.21 \pm 0.1$ & $3.65 \pm 0.5$ & $3.44 \pm 0.5$ & $3.74 \pm 0.0$ & $3.66 \pm 0.1$ & $3.32 \pm 0.1$ & & $3.45 \pm 0.2$ \\
\hline Residual & 0 & 0 & 1 & 0 & 2 & 5 & 2 & $3.26 \pm 0.51$ & 5 \\
\hline \multicolumn{10}{|l|}{$\quad$ Extracted } \\
\hline Metals & 16.32 & 16.09 & 15.98 & 17.43 & 17.04 & 16.49 & 16.2 & 16.82 & 15.78 \\
\hline Non-residual \% & 80.88 & 80.05 & 77.16 & 80.26 & 78.05 & 77.8 & 79.51 & 80.62 & 78.14 \\
\hline Residual \% & 19.12 & 19.95 & 22.84 & 19.74 & 21.95 & 22.2 & 20.49 & 19.38 & 21.86 \\
\hline Mobile phase $\%$ & 34.13 & 31.01 & 29.97 & 33.68 & 33.04 & 29.9 & 31.48 & 31.45 & 30.99 \\
\hline
\end{tabular}


Table 10: Summary of the metals $(\mathrm{mg} / \mathrm{Kg}$ ) in soils from Agricultural farmland

\begin{tabular}{|c|c|c|c|c|c|}
\hline Metals & Exchangeable F1 & Oxidizable F2 & Acid soluble F3 & Reducible F4 & Residual F5 \\
\hline $\mathrm{Cd}$ & ND & 0.22 & 0.36 & 0.21 & 0.41 \\
\hline $\mathrm{Cu}$ & 0.74 & 1.77 & 2.77 & 3.00 & 1.81 \\
\hline $\mathrm{Pb}$ & $\mathrm{ND}$ & 0.25 & 0.28 & 0.46 & 0.65 \\
\hline $\mathrm{Mn}$ & 11.23 & 8.98 & 10.74 & 9.74 & 20.93 \\
\hline $\mathrm{Co}$ & ND & 0.15 & 0.36 & 0.27 & 0.26 \\
\hline $\mathrm{Ni}$ & 1.14 & 1.37 & 1.29 & 2.40 & 1.05 \\
\hline $\mathrm{Zn}$ & 1.40 & 3.83 & 4.41 & 3.39 & 3.43 \\
\hline
\end{tabular}

Table 11: Percentage of metal associated with fractional forms in soils of Agricultural farmland in the study area.

\begin{tabular}{|l|l|l|l|l|l|}
\hline Metals & Exchangeable F1 & Oxidizible F2 & Acid soluble F3 & Reducible F4 & Residual F5 \\
\hline $\mathrm{Cd}$ & ND & 18.49 & 30.25 & 17.65 & 34.45 \\
\hline $\mathrm{Cu}$ & 7.33 & 17.54 & 27.45 & 29.73 & 17.94 \\
\hline $\mathrm{Pb}$ & $\mathrm{ND}$ & 15.24 & 17.07 & 28.05 & 39.63 \\
\hline $\mathrm{Mn}$ & 18.22 & 14.57 & 17.43 & 15.81 & 33.97 \\
\hline $\mathrm{Co}$ & $\mathrm{ND}$ & 14.42 & 34.62 & 25.96 & 2.00 \\
\hline $\mathrm{Ni}$ & 15.72 & 23.27 & 17.79 & 33.10 & 20.60 \\
\hline $\mathrm{Zn}$ & 8.51 & 26.79 & 20.00 & 20.84 \\
\hline
\end{tabular}

Table 12: Favourable nutrient supply of agricultural soils ( Odu et al., 1985)

\begin{tabular}{|l|l|}
\hline Elements & Critical values \\
\hline Organic matter & $2.6 \%$ \\
\hline Carbon & $1.513 \%$ \\
\hline Nitrogen & $0.15 \%$ \\
\hline Available phosphorus & $15 \mathrm{ppm}$ \\
\hline Calcium & $2.6 \mathrm{Me} / 100 \mathrm{~g}$ \\
\hline Potassium & $0.20 \mathrm{Me} / 100 \mathrm{~g}$ \\
\hline Magnesium & $0.04 \mathrm{Me} / 100 \mathrm{~g}$ \\
\hline $\mathrm{pH}$ & $6.5-7.5$ \\
\hline
\end{tabular}

Table13: Criteria for classification of soil property status (FAO Soil Bulletin 48)

\begin{tabular}{|l|l|l|l|}
\hline Parameters & Low value & Medium value & High value \\
\hline $\mathrm{pH}$ & $<5.6$ & $5.6-7.6$ & $>7.6$ \\
\hline Organic carbon $(\%)$ & $<0.8$ & $0.8-1.5$ & $>1.5$ \\
\hline CEC $(\mathrm{Me} / 100 \mathrm{~g})$ & $<16$ & $16-36$ & $>36$ \\
\hline Nitrogen $(\%)$ & $<0.83$ & $0.83-0.16$ & $>0.16$ \\
\hline Phosphorus $(\mathrm{Mg} / \mathrm{l})$ & $<6$ & $6-25$ & $>25$ \\
\hline Potassium $(\mathrm{Mg} / \mathrm{l})$ & $<140$ & $140-450$ & $>450$ \\
\hline Calcium $(\mathrm{Mg} / \mathrm{l})$ & $<1500$ & $1500-6000$ & $>6000$ \\
\hline Magnesium $(\mathrm{Mg} / \mathrm{l})$ & $<190$ & $190-550$ & $>550$ \\
\hline
\end{tabular}

\section{Discussion}

Physico-chemical Properties of Agricultural farmland soils.

\section{Soil pH}

The $\mathrm{pH}$ values ranged from 4.20-5.45. All the soils studied were acidic. These values are within those obtained by Onofiok and Ojobo, 1993; Rashad and Shalaby, 2007; Oguntimehin and Ipinmoroti, 2007; Onweremadu et al., 2007; Oviasoge and Ofomaja 2007; Osakwe and Akpoveta, 2012. They however differ from those suggested by Odu et al., 1985; Bamgbose et al., 2007. The pH of most mineral soils ranges from 5.5-7.5 (Radujevic and Bechkin, 1990). Radujevic and Bechkin, 1990, explained that acidic soils with pH 4.0-5.5 can have high concentrations of soluble aluminium and manganese ions which may be harmful to the growth of plants. Thus soil $\mathrm{pH}$ affects the availability of soil nutrients as well as heavy metals. Winterhalder (1984) stated that toxicity may occur if soil $\mathrm{pH}$ is below 5 and also reported that a $\mathrm{pH}$ of approximately 6-7 can release most readily available plant nutrients. This low $\mathrm{pH}$

(Acidity) of these farmland soils may be attributable to the presence of hydrogen cyanide from the main crop continuous cultivated on them. It may also be attributed to the high rainfall common in this area which could leach out basic cations from the farmland soils. The $\mathrm{pH}$ values obtained from this study revealed values lower than those recommended for agricultural soils (Odu et al., 1985).

The total Organic carbon; This is a measure of organic content in soils, sediments, and water (Yun, 2003). Lopez-Sanchez et al., 1996, observed that both anthropogenic natural processes have resulted in elevated concentrations of organic carbon in sediments. Organic carbon is the carbon stored in organic materials. It enters the soil through the decomposition of plants and animal residues, root exudates, living and dead micro- 
organisms and soil biota. Soil organic carbon is the main source of energy for soil micro-organisms. The percentage organic matter content of these soils ranged from $0.95-2.02 \%$, which is low but comparable with those obtained by Odoh et al., 2011; Osakwe and Akpoveta, 2012. This value is also lower than the critical levels required by soils for agricultural use as suggested by Odu et al., (1985). This suggests that these soils are poor and need some form of application of appropriate fertilizers. The reason for the low organic carbon could be due to the geology and the local parent material as demonstrated by Enwezor et al., (1981).

\section{Particle Size}

The soil particle size analysis showed the soil to be sandy. Clay ranged between $0.05-6.11 \%$, sand between 87.24-95.37\% and silt between 1.16-6.24\%.

\section{$\%$ Nitrogen and Phosphorus}

The percentage nitrogen ranged between $0.18-0.35 \%$. This value is comparable with the suggested $0.15 \%$ critical value by Odu et al., 1985. Available phosphorus ranged between $21.11-58.33 \mathrm{Mg} / \mathrm{Kg}$. This is within the recommended critical value of $2.6 \mathrm{Me} / 100 \mathrm{~g}$. The soils could be said to be rich in nitrogen and phosphorus. These farmland soils could be regarded as rich in Nitrogen and Phosphorus.

Cation Exchange Capacity (CEC). The cation exchange capacity was determined as the summation of cations $(\mathrm{Na}, \mathrm{K}, \mathrm{Mg}$, and $\mathrm{Ca})$. The overall cation exchange capacity ranged between 1.04-7.96 $\mathrm{Cmol} / \mathrm{kg}$. This low compared with the classification criteria of soil property (FAO Soil Bulletin 48). This low value may due to the inherent properties of the soil as derived from the local parent material. Akamigbo and Asadu (1986) demonstrated that the exchangeable cation and acidity of soils are controlled by the parent material from which the soil is derived. The heavy rainfall that is characteristic of this area may have promoted some level of leaching, which may probably be responsible for the low CEC.

\section{Sequential Extractions}

The sequential extraction scheme is a very useful tool in assessing the mobility and bioavailability of heavy metals in soils. The distribution of heavy metals in the soil samples allows us to predict their mobility, bioavailability and toxicity (Odoh et al., 2011). Fractionation of total metal contents may give indications of the origin of the metals. High levels in the exchangeable, acid soluble and reducible fraction may indicate pollution from anthropogenic origin, and even high contents in the resistant fractions except the residual fraction may be significant in the long term (Odoh et al., 2011). The results of sequential extraction of $\mathrm{Cd}, \mathrm{Cu}, \mathrm{Pb}, \mathrm{Mn}, \mathrm{Co}, \mathrm{Ni}$, and $\mathrm{Zn}$ in the agricultural farmland soils are presented in Table 5-9.

Cadmium (Cd); Total extracted cadmium ranged from $1.09-1.27 \mathrm{mg} / \mathrm{kg}$ which is below the critical permissible concentration of 3.0mg/kg (MAFF, 1992 and USEPA, 1986). The mobile phase contained 15.25-19.66\%. The metal can therefore not be said to be readily bioavailable

to the environment. The availability of this metal in the sequentially extracted fractions shows the order Residual> Acid soluble> Oxidizable> Reducible $>$ Exchangeable. The greatest percentage of $\mathrm{Cd}$ in these soils is the residual fraction with $34.45 \%$, followed by acid soluble $30.25 \%$, then oxidizable $18.49 \%$, reducible $17.65 \%$ and exchangeable ND.

Copper $(\mathbf{C u})$; The total extracted copper ranged from $8,47-12.16 \mathrm{mg} / \mathrm{kg}$. The levels of copper in all the sites studied were below the toxic limit of $250 \mathrm{mg} / \mathrm{kg}$ by USEPA (1986). The potential bioavailability of the metal is in the following order Reducible $(29.73 \%)>$ Acid soluble $(27.45 \%)>$ Residual (17.94) > Oxidizable (17.54\%) $>$ Extractable (7.33\%). The percentage in the mobile phase ranged from $16.95-35.77 \%$. This is appreciable and could be said to be readily bioavailable to the plants and to the environment. Copper is a micronutrient required by plants.

Lead $(\mathbf{P b})$ : The exchangeable lead level was not detected in all the farmland soil samples. The total extracted lead in the farmland soils ranged from $1.51-1.74 \mathrm{mg} / \mathrm{kg}$ with a mean of $1.64 \pm 0.07 \mathrm{mg} / \mathrm{kg}$. This level is below the permissible limits of $30-300 \mathrm{mg} / \mathrm{kg}$ (USEPA, 1986). These soils are therefore not polluted. The potential bioavailability of the metal is in the following order Residual $(39.63 \%)>$ Reducible $(28.05 \%)>$ Acid soluble $(17.07 \%)>$ Oxidizable $(15.24 \%)>$ Exchangeable (ND). Percentage of metal in mobile phase ranged from $12.35-18.96 \%$. This is low and is of minimal threat to the environment since it is below the allowed level. The highest percentage of the metal is in the residual fraction and so safe.

Manganese (Mn); The total extracted metal ranged from $19.75-23.20 \mathrm{mg} / \mathrm{kg}$. This value is within the permissible limit of $100-300 \mathrm{mg} / \mathrm{kg}$ (USEPA, 1986). The order of bioavailabilty is in the order Residual $(33.97 \%)>$ Exchangeable $(18.22 \%)>$ Acid Soluble $(17.43 \%)>$ Reducible $(15.81 \%)>$ Oxidizable $(14.57 \%)$. Percentage of metal in the mobile phase in the soils from the various agricultural farmland ranged from 30.55 $36.03 \%>$ this indicates that it quite available to the environment. 
Cobalt (Co); The metal was not detected in the exchangeable phase in all the farmland soils investigated. The total extracted metal ranged from $0.88-1.32 \mathrm{mg} / \mathrm{kg}$. The limit is not determined by the US EPA. The percentage of metal in the mobile phase ranged from $11.42-17.71 \%$. The order of bioavailabilty is Acid Soluble (34.62\%) $>$ Reducible $(25.96 \%)>$ Residual $(25.00 \%)>$ Oxidizable $(14.42 \%)>$ Exchangeable (ND). This metal is a micronutrient needed by plants.

Nickel (Ni); This metal presented in all the phases of the sequential extraction. The order of bioavailabilty is as follows; Reducible $(33.10 \%)>$ Oxidizable $(18.90 \%)>$ Acid Soluble $(17.79 \%)>$ Exchangeable $(15.72 \%)>$ Residual (14.48\%). The total extracted metal ranged from $7.08-7.97 \mathrm{mg} / \mathrm{kg}$ and the percentage in the mobile phase ranged from $28.53-40.70 \%$. Thus the metal is bioavailable to the plant.

Zinc (Zn); The total extracted metal ranged from $15.78-17.43 \mathrm{mg} / \mathrm{kg}$. The value is far below the USEPA (1986) permissible level of $300 \mathrm{mg} / \mathrm{kg}$. Percentage of metal in the mobile phase is appreciable (29.97- 34.13\%). The metal presented in all the phases of the sequential extraction; however an appreciable percentage is present in the mobile phase, making it readily available to plants. The order of bioavailabilty is; Acid Soluble (26.79) > Oxidizable (23.27) > Residual (20.84) > Reducible (20.60) > Exchangeable (8.51).

\section{References}

[1]. Afshin, M., Masoud, A. Z. (2008). Heavy Metals in Selected Edible Vegetable and Estimation of their Daily Intake in Sanandaj, Iran, Southeast Asia. J. Trop. Med. Public Health 39(2); 335-340.

[2]. Akamigbo, F. O. R., Asadu, C. L. A. (1986). The Influence of topography on the soil parameters in Selected areas of Anambra State, South-South Nigeria. Nig. J. Soil Sci. 6;35-46.

[3]. Albores, A. F., Perez-Cid, B., Cromes, E. F., Lopez, E. F. (2000). Comparison Between Sequential Extraction Procedures and Single Extraction Procedure for Metal Partioning in Sewage Sludge Samples. Analyst. 125; 1353-1357.

[4]. Bamgbose, O., Poeolu, B. O., Odukoya, O. O., Bamgbose, J. T., Olatunde, G. O.(2007). Physicochemical Characterization of leachate generated from simulated leaching of Refuse from Selected Waste Dumps in Abeokuta City, Nigeria. J. Chem. Soc. Nig. $22(1) ; 117-125$

[5]. Bougucos, G. J. (1962). Improved Hydrometer Method for Making Particle Size Analysis of Soils. Agron. J. 54;464-465

[6]. Duruibe, J. O., Oguwuegbu, M. O. C., Egwurugwu, J. N., (2007). Heavy Metal Pollution and Human Biotoxic Effects. Intern. J. Phys.Sci. 2(5):112-118.

[7]. Enwezor, W. O., Udo, E. J., Sobulo, R. A. (1981). Fertility Status and Productivity of acid sand of South Eastern, Nigeria. Soil Sci. $123 ; 110-116$

[8]. FAO (1988). Micro and Macro Nutrient Status. Bulletin 48.

[9]. Fergusson, J. E. (1990). The Heavy Element Chemistry; Environmental Impact and Health Effects. Pergamon Press, Oxford. 18-19, 27-40,55-79..

[10]. Hendershoh, W. H., Lalande, H., Duquette, M. (1993). Soil Reaction and Exchangeable Acidity. In: Carter, M. R (ed.), Soil Sampling and Methods of Analysis for Canadian Soc. Soil Sci. Lewis, Boca Raton, Fl 141-148.

[11]. Lemtech (2004). Water Treatment and Air Purification, Water Treatments, published by Enntech, Rotterdams Serge, Netherlands (www-excewater.com/hhp/filers/waterpurificationhtm). Retrieved 23/12/2007.

[12]. MAFF and Welch Office of Agriculture Department (1992). Code of good agricultural practice for the Protection of Soil Draft Consultation Document, MAFF, London.

[13]. Nriagu, J. O. (1989). Global Metal Poisoning: Poisoning the biosphere. Environment 32; 7-33.

[14]. Nwachukwu, M. A., Feng, H., Alinnor, J. (2010). Assessment of Heavy Metals Pollution in Soil and their Implications within and Around Mechanic Villages. Intern. J. Environ. Sci. Tech. 7(2); 347-358.

[15]. Odoh, R., Agbayi, E. B. , Kagbu, J. A., Thomas, S. A (2011). Heavy Metal Speciation in Agricultural Farmland in Some Selected Local Government Areas of Benue State, Nigeria. Arch. Appl. Sci. Res. 3 (3); 560-573.

[16]. Odu, C. T. I., Esuruoso, O. N., Oguwale (1985). In; Oghenero, O. A. (2012). Impact of degradation Processes on Physical and Chemical Properties of Soils in Delta State of the Niger Delta. J. Geol. Mining Res. 4(2) 13-22.

[17]. Oguntirnchin, I., Ipinmoroti, K (2008). Profile of Heavy Metals from Automobile Workshops in Akure, Nigeria. J. Environ. Sci. Tech. 1( 1); 19-26.

[18]. Onofiok, O. E., Ojobo, G. O. (1993). Inventory of Some Physico-chemical Characteristics of Some Nigerian Soils and their Relationship with Moisture Retention, Plasticity and Sealing Propensity. Samaru J. Agric. Res. 10; 15-27.

[19]. Onweremadu, E. U., Osuji, G. E., Eshett, E. T., Okpara, C. C., Ibeawuchi, I. I (2007). Characterization of Owner Manager Farm of Abia and Imo States for Sustainable Crop Production in South Eastern Nigeria. J. Ameri. Sci. 3 (1 ); 28-37.

[20]. Osakwe, S. A. (2010). Chemical Speciation and Mobility of Some Heavy Metals in Soils Around automobile waste Dumpsites in Northern Part of Niger Delta, South Central Nigeria. J. Appl. Sci. Environ. Manage. 14 (4); 123-130.

[21]. Osakwe, S. A., Akpoveta, V. O. (2012). Effect of cassava processing mill effluent on physical and chemical properties of soils in Abraka and Environs, Delta State, Nigeria. The Pacific J. Sci. Tech. 13 (2); 544-552.

[22]. Osakwe, S. A., Egharevba, F. (2008). Sequential Fractionation of Cadmium, Copper, Lead and Chromium in Soils around Municipal Solid Waste Dumps in Agbor, Nigeria. J. Chem. Soc. Nig. 33 (2); 139-147.

[23]. Oviasogie, P. O., Ofomaja, A. (2007). Available Mn, Zn, Fe, Pb and Physiocochemical changes associated with soil receiving cassava mill effluent. J. Chem. Soc. Nig. 32 (1);102-106.

[24]. Radujevic, M., Bechkin, (1990). Soil Sludge and Dust. Practical Environmental Analysis. The Royal Society of Chemistry. Cambridge. UK. 138-150, 303-313.

[25]. Rashad, M., Shalaby, F. A. (2007). Dispersion and Deposition of Heavy Metals around Municipal Solid Waste (MSW) Dumpsites, Alexandria, Egypt. American-Eurasian J. Agric. Environ. Sci. 85;292-294.

[26]. Tack, F. M. G., Verloo, M. G (1995). Chemical Speciation and Fractionation in soil and sediment heavy metal analysis; a review, Intern. J Environ. Anal. Chem. 59:225-238.

[27]. Tan, K. M. (1996). Soil Sampling Preparation and Analysis. Mercel Dekkes Inc. New York. Basel. Hong Kong. 
[28]. Tessier, A., Campbell, P. G. C., Bisson, M. (1979). Sequential Extraction Procedure for the Speciation of Particulate Trace Metals. Analy. Chem. 51 (7); 844-851.

[29]. Tsafe, A. I., Hassan, L. G., Sahabi, D. M., Alhassan, Y., Bala, B. M. (2012). Evaluation of Heavy Metals Uptake and Risk Assessment of Vegetables Grown in Yargalma of Northern Nigeria. J. Basic and App. Sci. Res. 2 (7); 6708-6714.

[30]. Uba, S., Uzairu, A., Okunola, O. J. (2009). Content of Metals in Lumbricus terrestries and Associated Soils in Dumpsites. Intern. J. Environ. Res. 3 (3):353-358.

[31]. USEPA (1986). Test Methods of Evaluation for Solid Waste ( USEPA S/W 846 USEPA Washington DC).

[32]. Walkley, A., Black, I. A. (1934). An Examination of the Detjare Method for Determining Soil Organic Matter and a Proposed Modification of Chromic acid titration. Soil sci. 37;29-36.

[33]. Winterhalder, 1984, In: Zaku, S. G., Emmanual, S. A., Thomas, S. A. (2011). Assessing the level of soil nutrients; a case study of Donga, Ibi and Wakari Farmlands in Taraba State, Nigeria. Agric. J. N. Am. 2 (1); 101-108.

[34]. Yun, O (2003). Simlation Dynamic Load of Naturally occurring Total Organic Carbon (TOC) from watershed. Water Research. 5537; 823-832. 